\title{
Optic disc drusen and episodic visual loss
}

\author{
N J C SARKIES AND M D SANDERS \\ From the Department of Ophthalmology, the National Hospital for Nervous Diseases, London
}

SUMMARY A case is reported in which recurrent episodes of visual loss occurred over a period of 26 years in a patient with bilateral optic disc drusen. Visual field loss was associated with episodes of ischaemic optic neuropathy. The possible mechanism is discussed.

Optic disc drusen may be associated with arcuate scotomas, constriction of the peripheral visual field or rarely the loss of central vision. The progression of visual field defects is usually insidious.' There are reports of ischaemic optic neuropathy occurring with drusen, but these have been solitary events. ${ }^{23}$ This report documents recurrent episodes of visual loss occurring over a period of 26 years in a patient with optic disc drusen.

\section{CASE REPORT}

The patient was a 54-year-old right handed woman with a history of recurrent episodes of painless visual loss.

She first presented to the National Hospital in 1966 with a seven-year history of field loss in her left eye and a 10-day history of blurred vision in her right eye.

A general examination gave normal results. An ophthalmic examination found visual acuities right

Correspondence to M D Sanders, FRCS, Department of NeuroOphthalmology, National Hospital for Nervous Diseases, Queen Square, London WC1N 3BG.

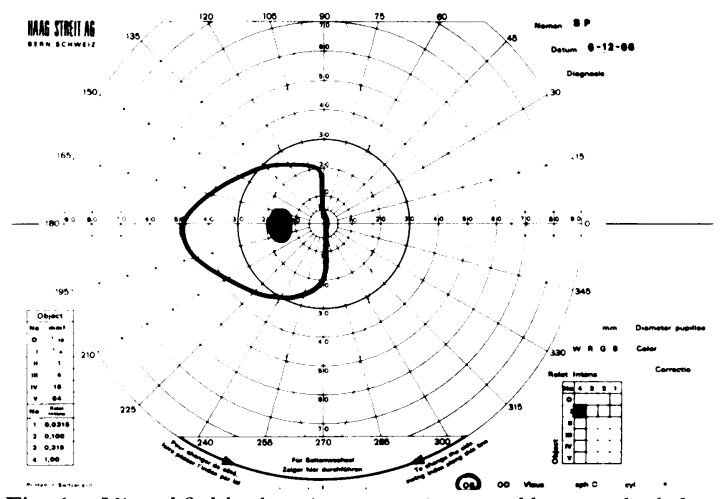

$6 / 6$ and left $6 / 24$. The visual fields showed extensive nasal loss on the left side and a full right field (Fig. 1). Nerve fibre loss was noted on the left disc, and there were optic disc drusen. On the right disc there were buried drusen and the inferior part of the disc was swollen with distended neurones, cotton-wool spots, and superficial haemorrhages.

$X$-rays of the skull and optic foramina, bilateral carotid angiograms, and an air ventriculogram were normal.

In $1970,1976,1978$, and 1981 she had recurrent episodes of visual loss and on each occasion she was found to have optic disc swelling. Treatment with corticosteroids apparently improved visual function. She was first seen by one of us in 1981. Visual acuities were right $6 / 18$ and left $6 / 60$. Visual fields showed extensive loss on the left and diffuse constriction on the right. There were drusen superonasally on the right disc and numerous drusen on the left. A computed tomographic scan of the orbit and brain showed no intracranial lesion and normal sized optic nerves (Fig. 2). It was concluded that the disc drusen

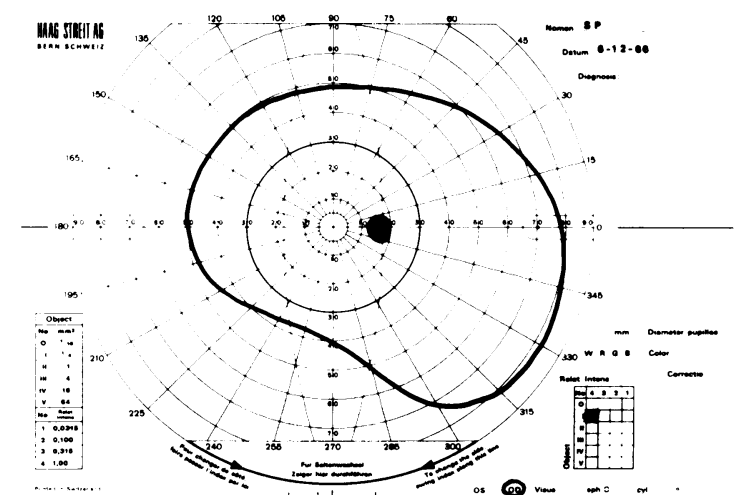

Fig. 1 Visual fields showing extensive nasal loss on the left and normal right field. 

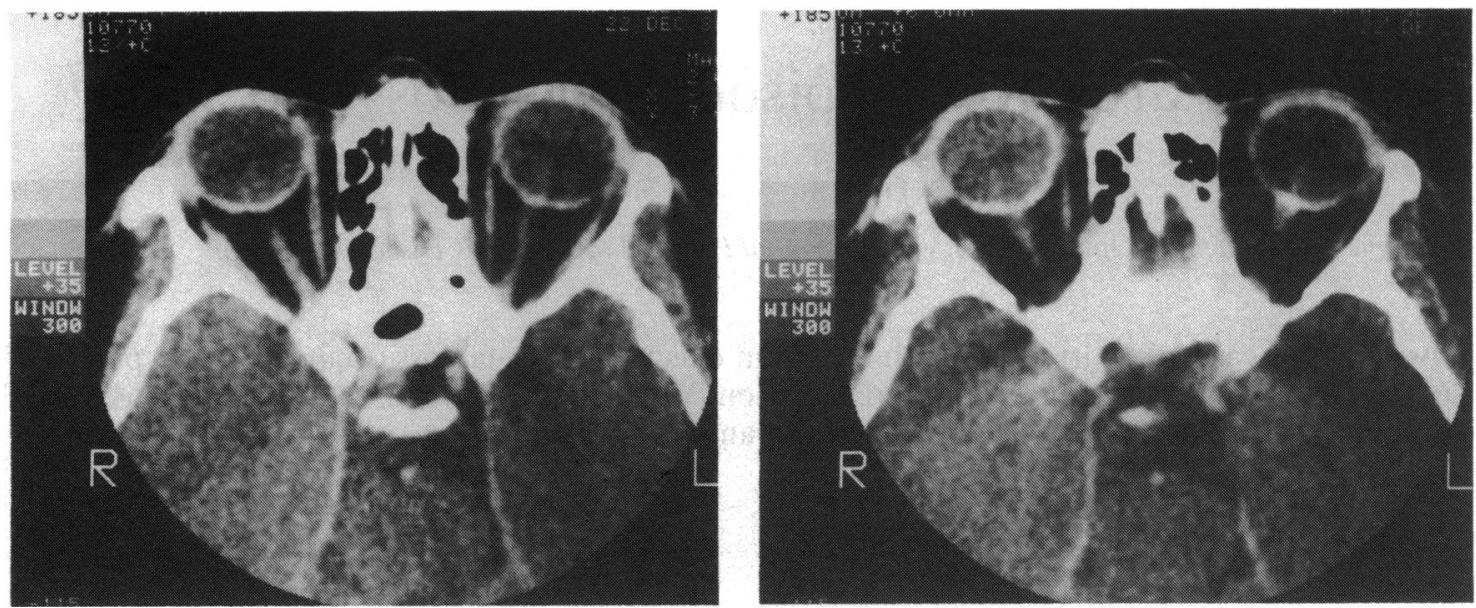

Fig. 2 Computerised tomographic scan of orbit showing normal optic nerves, and left drusen.

were associated with recurrent episodes of ischaemic swelling of the optic nerves.

Over the next four years she had three further episodes of visual loss, in the right eye in 1984 and 1985 and in the left eye in 1985 . She was treated with corticosteroids during each episode.

When seen by us in December 1985 visual acuities were right $6 / 9$, left counting fingers. Further constriction of the visual fields had occurred (Fig. 3). Examination of the discs showed bilateral optic disc drusen (Fig. 4).

\section{Discussion}

Optic disc drusen consist of calcified, laminated aggregates of extracellular material. Recent ultrastructural studies by $\mathrm{Tso}^{4}$ and Spencer ${ }^{5}$ have indicated that drusen form as a result of alterations in axoplasmic transport at the disc producing accumulations of axoplasmic material.

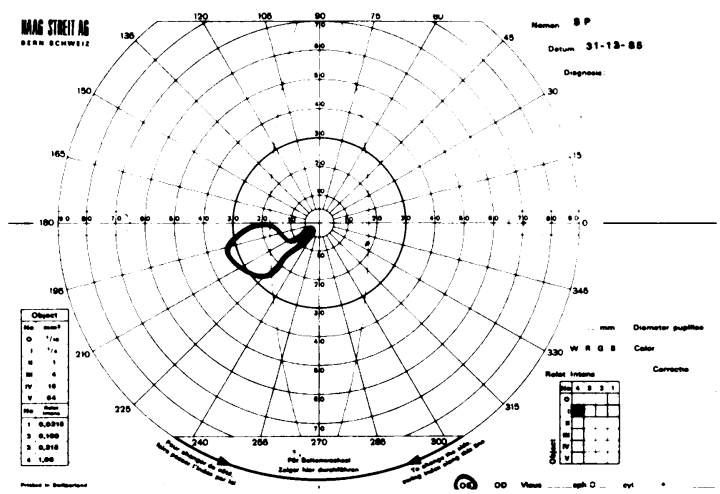

Fig. 3 Visual fields showing constriction of both visual fields.
The association between optic disc drusen and visual field defects is common. In a large study Lorentzen found field defects in $87 \%$ patients with drusen, ${ }^{6}$ but it is rare for optic disc drusen to be associated with loss of central vision, and it has been argued that this occurrence should prompt further investigation for another cause.'

The mechanism by which optic disc drusen produce field loss is uncertain. The visual field defects do not correspond to the position of the drusen on the disc. The acute onset of field defects suggests a vascular event. Circulation of the disc may be disturbed by optic disc drusen, because the vascular supply is anomalous, predisposing towards optic disc haemorrhage or ischaemic optic neuropathy. Optic disc drusen are commonly found in discs with an anomalous vascular pattern, especially an increased number of arterial or venous branches. ${ }^{7}$ Several reports have drawn attention to the occurrence of haemorrhage and ischaemic optic neuropathy with

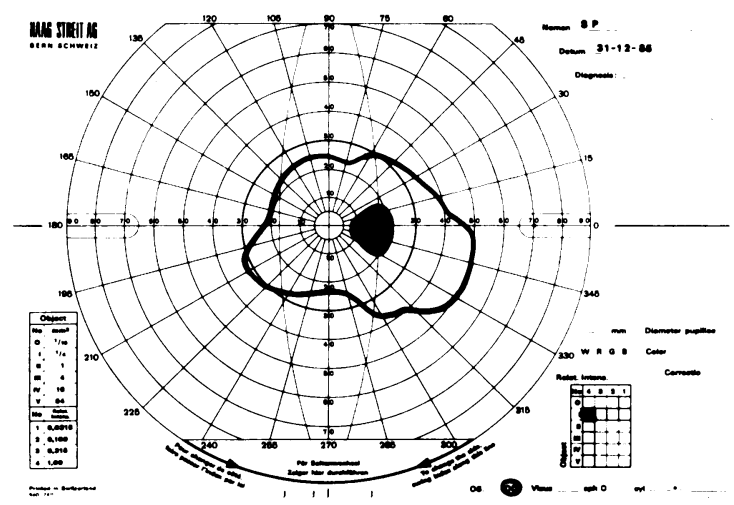




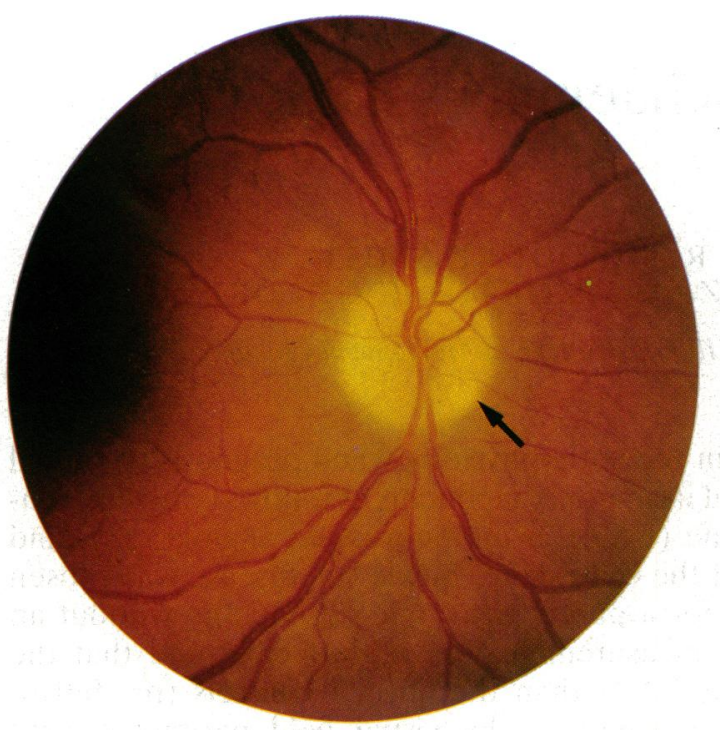

Fig. 4 Optic discs showing disc drusen (arrowed).

arcuate field loss and segmental disc pallor. This has been noted in four patients with optic disc drusen. ${ }^{8}$

The circulation of the disc with drusen may also be more vulnerable because the disc is small. Recently an association has been established between optic disc drusen and an abnormally small disc. ${ }^{9}$ It is well recognised that there is an increased likelihood of ischaemic optic neuropathy occurring in small discs."

In our patient with optic disc drusen it is probable that the recurrent episodes of visual loss were due to periods of relative vascular insufficiency producing ischaemic optic neuropathy. The rationale for treatment with corticosteroids during each episode of visual loss was to reduce additional damage caused by neuronal swelling. ${ }^{1}$ Firm evidence is lacking that this treatment improved visual function, but it is of interest that she still retained $6 / 9$ vision in one eye despite numerous episodes of visual loss.

We are indebted to Miss Josephine Lace for secretarial assistance and Mr Richard Dewhirst for the illustrations.

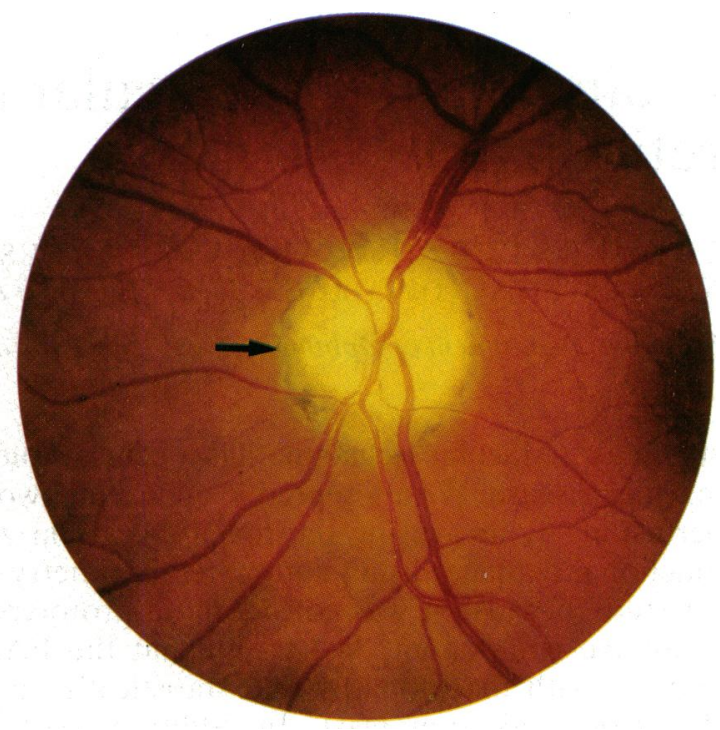

References

1 Knight CL, Hoyt WF. Monocular blindness from drusen of the optic disk. Am J Ophthalmol 1972; 73: 890-2.

2 Cohen DN. Drusen of the optic disc and the development of field defects. Arch Ophthalmol 1971; 85: 224-6.

3 Karel I, Otradovec J, Peleska M. Fluorescein angiography in circulatory disturbances in drusen of the optic disc. Ophthalmologica 1972; 164: 449-62.

4 Tso MOM. Pathology and pathogenesis of drusen of the optic nerve head. Ophthalmology 1981; 88: 1066-80.

5 Spencer WH. Drusen of the optic disk and aberrant axoplasmic transport. The XXXIV Edward Jackson Memorial Lecture. Am J Ophthalmol 1978; 85: 1-12.

6 Lorentzen DW. Drusen of the optic disk. Acta Ophthalmol (Kbh) 1966; 1 (suppl 90): 179-84.

7 Erkkila $\mathrm{H}$. The central vascular pattern of the eye ground in children with drusen of the optic disc. Graefes Arch Clin Exp Ophthalmol 1976; 199: 1-10.

8 Sanders TE, Gay AJ, Newman M. Hemorrhagic complications of drusen of the optic disk. Am J Ophthalmol 1971; 71: 204-17.

9 Mullie MA, Sanders MD. Scleral canal size and optic nerve head drusen. Am J Ophthalmol 1984; 99: 356-9.

10 Beck RW, Savino PJ, Repka MX, Schatz NJ, Sergott RC. Optic disc structure in anterior ischaemic optic neuropathy. Ophthalmology 1984; 9: 1334-7.

11 Shults WT. Ischemic optic neuropathy: still the ophthalmologist's dilemma. Ophthalmology 1984: 91: 1338-41. 